Linearization of Lagrange and Hermite interpolating matrix polynomials

Roel Van Beeumen

Wim Michiels

Karl Meerbergen

Report TW627, May 2013

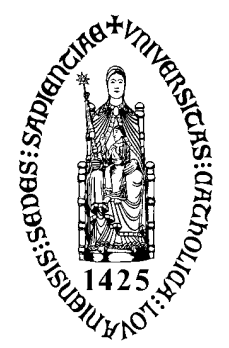

KU Leuven 


\title{
Linearization of Lagrange and Hermite interpolating matrix polynomials
}

\author{
Roel Van Beeumen \\ Wim Michiels \\ Karl Meerbergen \\ Report TW627, May 2013 \\ Department of Computer Science, KU Leuven
}

\begin{abstract}
This paper considers interpolating matrix polynomials $P(\lambda)$ in Lagrange and Hermite bases. A classical approach to investigate the polynomial eigenvalue problem $P(\lambda) x=0$ is linearization, by which the polynomial is converted into a larger matrix pencil with the same eigenvalues. Since the current linearizations of degree $n$ Lagrange polynomials consist of matrix pencils with $n+2$ blocks, they introduce additional eigenvalues at infinity. Therefore, we introduce new linearizations which overcome this. Initially, we restrict to Lagrange and barycentric Lagrange matrix polynomials and give two new and more compact linearizations, resulting in matrix pencils of $n+1$ and $n$ blocks for polynomials of degree $n$. For the latter, there is a one-to-one correspondence between the eigenpairs of $P(\lambda)$ and the eigenpairs of the pencil. We also prove that these linearizations are strong. Moreover, we show how to exploit the structure of the proposed matrix pencils in Krylov-type methods, so that in this case we only have to deal with linear system solves of matrices of the original matrix polynomial dimension. Finally, we generalize for multiple interpolation and introduce new linearizations for Hermite Lagrange and barycentric Hermite matrix polynomials. Again, we can show that the linearizations are strong and that there is a one-to-one correspondence of the eigenpairs.
\end{abstract}

Keywords : matrix polynomials, matrix pencil, linearization, strong linearization, Lagrange interpolation, Hermite interpolation, barycentric form.

MSC : 65F15, 65D05, 15A22. 


\title{
Linearization of Lagrange and Hermite interpolating matrix polynomials*
}

\author{
Roel Van Beeumen ${ }^{\dagger} \quad$ Wim Michiels $^{\dagger} \quad$ Karl Meerbergen $^{\dagger}$
}

May 12, 2013

\begin{abstract}
This paper considers interpolating matrix polynomials $P(\lambda)$ in Lagrange and Hermite bases. A classical approach to investigate the polynomial eigenvalue problem $P(\lambda) x=0$ is linearization, by which the polynomial is converted into a larger matrix pencil with the same eigenvalues. Since the current linearizations of degree $n$ Lagrange polynomials consist of matrix pencils with $n+2$ blocks, they introduce additional eigenvalues at infinity. Therefore, we introduce new linearizations which overcome this. Initially, we restrict to Lagrange and barycentric Lagrange matrix polynomials and give two new and more compact linearizations, resulting in matrix pencils of $n+1$ and $n$ blocks for polynomials of degree $n$. For the latter, there is a one-to-one correspondence between the eigenpairs of $P(\lambda)$ and the eigenpairs of the pencil. We also prove that these linearizations are strong. Moreover, we show how to exploit the structure of the proposed matrix pencils in Krylov-type methods, so that in this case we only have to deal with linear system solves of matrices of the original matrix polynomial dimension. Finally, we generalize for multiple interpolation and introduce new linearizations for Hermite Lagrange and barycentric Hermite matrix polynomials. Again, we can show that the linearizations are strong and that there is a one-to-one correspondence of the eigenpairs.
\end{abstract}

Keywords: matrix polynomials; matrix pencil; linearization; strong linearization; Lagrange interpolation; Hermite interpolation; barycentric form

AMS subject classifications: 65F15, 65D05, 15A22

\section{Introduction}

The original Lagrange form, introduced in [10], has certain shortcomings, e.g., increasing the degree of the polynomial by adding a new interpolation point requires computations from scratch and also the computation is numerically unstable [3]. Therefore, Lagrange interpolation is frequently considered as a bad choice for practical computations and thus mainly an analytic or theoretical tool for proving theorems. Nevertheless, rewriting in the so called modified Lagrange form and the barycentric Lagrange form overcomes the shortcomings of the original form and makes Lagrange interpolation very suitable for practical use.

In this paper, we consider matrix polynomials in Lagrange and Hermite bases. Generally, for every polynomial basis, an interpolating matrix polynomial $P(\lambda) \in \mathbb{C}^{s \times s}$ of

* This work has been supported by the Programme of Interuniversity Attraction Poles of the Belgian Federal Science Policy Office (IAP P6-DYSCO), by OPTEC, the Optimization in Engineering Center of the KU Leuven, by the projects STRT1-09/33 and OT/10/038 of the KU Leuven Research Council and the project G.0712.11N of the Research Foundation-Flanders (FWO).

$\dagger^{\dagger}$ Department of Computer Science, KU Leuven, University of Leuven, 3001 Heverlee, Belgium (Roel.VanBeeumen@cs.kuleuven.be,Wim.Michiels@cs.kuleuven.be, Karl.Meerbergen@cs.kuleuven.be). 
degree $n$ is uniquely determined by $n+1$ samples of the function $A_{i}:=P\left(\sigma_{i}\right)$, where $\sigma_{i} \in \mathbb{C}, i=0,1, \ldots, n$ are distinct interpolation points. The polynomials $P(\lambda)$ in modified or barycentric Lagrange form can be constructed very easily, since we immediately use these function values $A_{i}$ in combination with the barycentric weights, which are computed from the interpolation points $\sigma_{i}$. However, for several point distributions we have explicit formulas for these barycentric weights. This is in contrast to Newton's interpolation, where divided differences have to be computed from the function values $A_{i}$. Also for Chebyshev interpolation coefficient matrices have to be computed.

Polynomial eigenvalue problems (PEPs): $P(\lambda) x=0$, where $P(\lambda)$ is a complex $s \times$ $s$ matrix polynomial of degree $n$ in $\lambda$ and $x \in \mathbb{C}^{s} \backslash\{0\}$, occur in a wide number of applications, e.g., vibration analysis of buildings and machines. The classical and most common approach to solve PEPs is linearization, i.e., we mean the conversion of $P(\lambda) x=$ 0 into a larger size linear eigenvalue problem $L(\lambda) y=\left(C_{0}-\lambda C_{1}\right) y=0$ with the same eigenvalues. This linear eigenvalue problem can then be solved by standard techniques.

Linearization is also a commonly used technique for solving nonlinear eigenvalue problems. In the last decade, several linearizations for different polynomial bases are proposed in the literature. See, e.g., [9] for monomial basis, [6, 9] for Chebyshev basis and [15] for Newton basis. However, to the authors' knowledge, Lagrange basis was never used. A possible explanation is that the current linearizations for matrix polynomials in Lagrange basis $[1,2]$ and also in Hermite Lagrange basis [14] contain more eigenvalues than the original polynomial $P(\lambda)$ by introducing additional eigenvalues at infinity.

Therefore, we will now propose new linearizations for the Lagrange and barycentric Lagrange polynomial which ensure a one-to-one correspondence between the eigenpairs of $P(\lambda)$ and the eigenpairs of the pencil obtained after linearization. We also generalize for multiple interpolation and introduce new linearizations for the Hermite Lagrange and barycentric Hermite polynomial.

This paper is organized as follows. Section 2 introduces some basic definitions and notation. Section 3 reviews Lagrange interpolation and the derivation of the barycentric Lagrange form. In Section 4 we reformulate the linearization of the Lagrange and barycentric Lagrange polynomial of dimension $(n+2) s$ and introduce two new linearizations of dimension $(n+1) s$ and $n s$. We also prove for the last one that this is a strong linearization. In Section 5 we illustrate how the structure of the proposed pencil can be exploited in Krylov-type methods. Section 6 reviews the Hermite interpolating Lagrange and barycentric matrix polynomial. In Section 7 we generalize the linearizations of Section 4 for multiple interpolation. Finally, the main conclusions are summarized in Section 8 .

\section{Definitions and notation}

We study linearizations of matrix polynomials $P(\lambda) \in \mathbb{C}^{s \times s}$, where $P(\lambda)$ is regular, i.e., $\operatorname{det} P(\lambda)$ does not vanish identically. Linearization is the classical approach for investigating and solving PEPs. In this case, matrix polynomials are transformed into linear matrix pencils with the same eigenvalues. Therefore, unimodular matrix polynomials are used, i.e., matrix polynomials $E(\lambda)$ such that $\operatorname{det} E(\lambda)$ is a nonzero constant and independent of $\lambda$. We now introduce some basic definitions and notation in order to support the elaboration in the remaining sections.

Definition 2.1 (Weak linearization [7]). Let $P(\lambda)$ be an $s \times s$ matrix polynomial of degree $n$ with $n \geq 1$. A pencil $L(\lambda)=\left(C_{0}-\lambda C_{1}\right)$ with $C_{0}, C_{1} \in \mathbb{C}^{n s \times n s}$ is called a linearization of $P(\lambda)$ if there exist unimodular matrix polynomials $E(\lambda), F(\lambda)$ such that

$$
E(\lambda) L(\lambda) F(\lambda)=\left[\begin{array}{cc}
P(\lambda) & 0 \\
0 & I_{(n-1) s}
\end{array}\right] .
$$


In order to allow $\infty$ as a possible eigenvalue of $P(\lambda)$ we introduce the definition of the reversal of matrix polynomials and of a strong linearization.

Definition 2.2 (Reversal of matrix polynomials). For a matrix polynomial $P(\lambda)$ of degree $n$, the reversal of $P(\lambda)$ is the polynomial $P^{\#}(\lambda):=\lambda^{n} P\left(\lambda^{-1}\right)$.

Note that the nonzero finite eigenvalues of $P^{\#}(\lambda)$ are the reciprocals of those of $P(\lambda)$ and that an eigenvalue at $\infty$ of $P(\lambda)$ corresponds to an eigenvalue 0 of the reversal polynomial $P^{\#}(\lambda)$.

Definition 2.3 (Strong linearization [11]). An ns $\times$ ns linear matrix pencil $C_{0}-\lambda C_{1}$ is a strong linearization of the $s \times s$ regular matrix polynomial $P(\lambda)$ of (possibly extended) degree $n$ if there are unimodular matrix polynomials $E(\lambda)$ and $E(\lambda)$ such that

$$
\left[\begin{array}{cc}
P(\lambda) & 0 \\
0 & I_{(n-1) s}
\end{array}\right]=E(\lambda)\left(C_{0}-\lambda C_{1}\right) F(\lambda),
$$

and there are unimodular matrix polynomials $H(\lambda)$ and $K(\lambda)$ such that

$$
\left[\begin{array}{cc}
P^{\#}(\lambda) & 0 \\
0 & I_{(n-1) s}
\end{array}\right]=H(\lambda)\left(\lambda C_{0}-C_{1}\right) K(\lambda) .
$$

The following theorem gives conditions for a (strong) linearization, which will be used in the subsequent analysis. It is based on the local Smith form.

Theorem 2.4. [11] Let $P(\lambda)$ be an $s \times s$ regular matrix polynomial of extended degree $n$ and let $C_{0}-\lambda C_{1}$ be an $n s \times n s$ linear matrix function. Assume that, for each distinct finite eigenvalue $\lambda_{i}$, there exist functions $E_{i}(\lambda)$ and $F_{i}(\lambda)$ which are unimodular and analytic in a neighbourhood of $\lambda_{i}$ and for which

$$
\left[\begin{array}{cc}
P(\lambda) & 0 \\
0 & I_{(n-1) s}
\end{array}\right]=E_{j}(\lambda)\left(C_{0}-\lambda C_{1}\right) F_{j}(\lambda),
$$

then $C_{0}-\lambda C_{1}$ is a (weak) linearization of $P(\lambda)$.

If $P^{\#}(\lambda)$ has an eigenvalue at zero, assume also that there are functions $E_{0}(\lambda)$ and $F_{0}(\lambda)$ which are unimodular and analytic in a neighbourhood of $\lambda=0$ and for which

$$
\left[\begin{array}{cc}
P^{\#}(\lambda) & 0 \\
0 & I_{(n-1) s}
\end{array}\right]=E_{0}(\lambda)\left(\lambda C_{0}-C_{1}\right) F_{0}(\lambda),
$$

then $C_{0}-\lambda C_{1}$ is a strong linearization of $P(\lambda)$.

\section{Lagrange interpolation}

In this section, we review the interpolating Lagrange matrix polynomial. We start with the original form, followed by the modified form and end with the barycentric form.

\subsection{Original Lagrange form}

Suppose an $s \times s$ matrix function $A(\lambda)$ is sampled at $n+1$ distinct interpolation points (nodes) $\sigma_{i}, i=0, \ldots, n$, with corresponding values $A_{i}:=A\left(\sigma_{i}\right)$. The Lagrange interpolation problem addressed here is that of finding the $s \times s$ matrix polynomial $P(\lambda)$, of degree at most $n$, such that $P$ interpolates $A$ at the points $\sigma_{i}$, i.e.,

$$
P\left(\sigma_{i}\right)=A_{i}, \quad i=0, \ldots, n .
$$


This problem is well-posed and the solution can be written in Lagrange form [10]:

$$
P(\lambda)=\sum_{i=0}^{n} A_{i} \ell_{i}(\lambda)
$$

where the Lagrange polynomials $\ell_{i}(\lambda)$ are defined as

$$
\ell_{i}(\lambda)=\frac{\prod_{k=0, k \neq i}^{n}\left(\lambda-\sigma_{k}\right)}{\prod_{k=0, k \neq i}^{n}\left(\sigma_{i}-\sigma_{k}\right)}, \quad i=0, \ldots, n,
$$

with the following property at the nodes

$$
\ell_{i}\left(\sigma_{k}\right)=\left\{\begin{array}{ll}
1, & i=k, \\
0, & \text { otherwise },
\end{array} \quad i, k=0, \ldots, n .\right.
$$

\subsection{Modified Lagrange form}

The original Lagrange formula (3.1) can be rewritten in such a way that it can be evaluated and updated in $O(n)$ operations [3]. Therefore, note that the numerator of $\ell_{i}$ in (3.2) can be written as the polynomial

$$
\ell(\lambda)=\left(\lambda-\sigma_{0}\right)\left(\lambda-\sigma_{1}\right) \cdots\left(\lambda-\sigma_{n}\right)
$$

divided by $\lambda-\sigma_{i}$. Defining the barycentric weights by

$$
w_{i}=\frac{1}{\prod_{k \neq i}\left(\sigma_{i}-\sigma_{k}\right)}, \quad i=0, \ldots, n,
$$

that is, $w_{i}=1 / \ell^{\prime}\left(\sigma_{i}\right)$, allows us to write $\ell_{i}$ as

$$
\ell_{i}(\lambda)=\ell(\lambda) \frac{w_{i}}{\lambda-\sigma_{i}}, \quad i=0, \ldots, n .
$$

Now, note that all terms of the sum in (3.1) contain the factor $\ell(\lambda)$, which is independent of $i$. Bringing this factor in front of the sum yields the modified Lagrange form [3]:

$$
P(\lambda)=\ell(\lambda) \sum_{i=0}^{n} A_{i} \frac{w_{i}}{\lambda-\sigma_{i}}
$$

This modified Lagrange form (3.6) is shown to be backward stable [8].

\subsection{Barycentric Lagrange form}

The modified Lagrange form (3.6) can still be modified to an even more elegant form which is often used in practice. We start from

$$
1=\sum_{i=0}^{n} \ell_{i}(\lambda)=\ell(\lambda) \sum_{i=0}^{n} \frac{w_{i}}{\lambda-\sigma_{i}} .
$$

Dividing the modified Lagrange form for $P(\lambda)$ (3.6) by (3.7) and cancelling out the common factor $\ell(\lambda)$, we obtain the barycentric form [3]:

$$
P(\lambda)=\frac{\sum_{i=0}^{n} A_{i} \frac{w_{i}}{\lambda-\sigma_{i}}}{\sum_{i=0}^{n} \frac{w_{i}}{\lambda-\sigma_{i}}}=\sum_{i=0}^{n} A_{i} b_{i}(\lambda),
$$


where

$$
b_{i}(\lambda)=\frac{1}{b(\lambda)} \cdot \frac{w_{i}}{\lambda-\sigma_{i}}, \quad i=0, \ldots, n,
$$

with

$$
b(\lambda)=\sum_{i=0}^{n} \frac{w_{i}}{\lambda-\sigma_{i}} .
$$

The barycentric form is a Lagrange form, but one with a special symmetry. The weights $w_{i}$, still defined by (3.4), appear in the denominator exactly as in the numerator, except without the data factors $A_{i}$. Therefore, any common factor in all the weights $w_{i}$ can be cancelled without affecting the value $P$.

Like the modified Lagrange form, the barycentric one also takes advantage of updating the weights $w_{i}$ in $O(n)$ flops to incorporate a new data pair $\left(\sigma_{n+1}, A_{n+1}\right)$. In [8] it is proved that the barycentric Lagrange interpolation form is forward stable for any set of interpolating points with a small Lebesgue constant. Finally, note that even if other weights $w_{i}$ than (3.4) would be chosen in (3.8), the resulting rational function would still interpolate at the nodes $\sigma_{i}$ in the sense that $P\left(\sigma_{i}\right)=A_{i}$.

\section{Linearization of the Lagrange and barycentric La- grange polynomial}

To the authors' knowledge, a linearization in a companion pencil of the Lagrange polynomial was first introduced by Amiraslani in [1]. Firstly in $\S 4.1$, we review this linearization of dimension $(n+2) s$ in a slightly different form and extend it also to the barycentric Lagrange polynomial. Next, we introduce two new and more compact linearizations of dimension $(n+1) s$ and $n s$ in $\S 4.2$ and $\S 4.3$, respectively.

\subsection{Linearization of dimension $(n+2) s$}

The companion pencil of the Lagrange polynomial, introduced in $[1,2]$, can be extended to the barycentric Lagrange polynomial. Therefore, we review this linearization in a slightly different form by the following theorem.

Theorem 4.1. Let $P(\lambda) \in \mathbb{C}^{s \times s}$ be a matrix polynomial of degree $n$ in modified Lagrange form (3.6) or in barycentric Lagrange form (3.8). Then, the $(n+2) s \times(n+2)$ s linear companion pencil

$$
L(\lambda)=C_{0}-\lambda C_{1},
$$

where

$$
C_{0}=\left[\begin{array}{ccccc}
A_{0} & A_{1} & \cdots & A_{n} & 0 \\
\sigma_{0} I & & & & w_{0} I \\
& \sigma_{1} I & & & w_{1} I \\
& & \ddots & & \vdots \\
& & & \sigma_{n} I & w_{n} I
\end{array}\right], \quad C_{1}=\left[\begin{array}{ccccc}
0 & & & & \\
I & 0 & & & \\
& I & 0 & & \\
& & \ddots & \ddots & \\
& & & I & 0
\end{array}\right],
$$

is a linearization of $P(\lambda)$.

In order to illustrate the construction of the pencil in Theorem 4.1, we define $\underline{\Lambda}(\lambda)$ 
and $\underline{B}(\lambda)$ as follows

$$
\underline{\Lambda}(\lambda)=\left[\begin{array}{c}
\ell_{0}(\lambda) \\
\ell_{1}(\lambda) \\
\vdots \\
\ell_{n}(\lambda) \\
\ell(\lambda)
\end{array}\right] \quad \text { and } \quad \underline{B}(\lambda)=\left[\begin{array}{c}
b_{0}(\lambda) \\
b_{1}(\lambda) \\
\vdots \\
b_{n}(\lambda) \\
b(\lambda)^{-1}
\end{array}\right]
$$

Note that $\underline{\Lambda}(\lambda)=\ell(\lambda) b(\lambda) \underline{B}(\lambda)$. Then, following the notation of [12], we have for the Lagrange polynomial (3.6)

$$
\left(C_{0}-\lambda C_{1}\right)(\underline{\Lambda}(\lambda) \otimes I)=e_{1} \otimes P(\lambda),
$$

and similarly for the barycentric Lagrange polynomial (3.8)

$$
\left(C_{0}-\lambda C_{1}\right)(\underline{B}(\lambda) \otimes I)=e_{1} \otimes P(\lambda),
$$

where the product of the first block row of $C_{0}-\lambda C_{1}$ with $\underline{\Lambda}(\lambda) \otimes I$ and $\underline{B}(\lambda) \otimes I$, respectively, is the matrix polynomial $P(\lambda)$. The remaining products simply reproduce the relations (3.5) and (3.9), respectively.

Corollary 4.2. Suppose that $\left(\lambda_{\star}, x\right)$ is an eigenpair of $P(\lambda)$ and that $L(\lambda)=C_{0}-\lambda C_{1}$ is defined by Theorem 4.1. Then, evaluating (4.2) and (4.3) at $\lambda_{\star}$ and multiplying to the right by $x$ yields

$$
L\left(\lambda_{\star}\right) \cdot\left(\underline{\Lambda}\left(\lambda_{\star}\right) \otimes x\right)=L\left(\lambda_{\star}\right) \cdot\left(\underline{B}\left(\lambda_{\star}\right) \otimes x\right)=0 .
$$

Thus, $\lambda_{\star}$ is also an eigenvalue of $L(\lambda)=C_{0}-\lambda C_{1}$ with the corresponding structured eigenvector $\underline{\Lambda}\left(\lambda_{\star}\right) \otimes x$ or $\underline{B}\left(\lambda_{\star}\right) \otimes x$, since $\underline{\Lambda}\left(\lambda_{\star}\right)=\ell\left(\lambda_{\star}\right) b\left(\lambda_{\star}\right) \underline{B}\left(\lambda_{\star}\right)$. of

In [2] it is proven that the pencil $C_{0}-\lambda C_{1}$, as defined in (4.1), is a strong linearization

$$
\underline{\widehat{P}}(\lambda):=\lambda^{n+2} 0_{s}+\lambda^{n+1} 0_{s}+P(\lambda) .
$$

\subsection{Linearization of dimension $(n+1) s$}

We now propose a new linearization for the Lagrange and barycentric Lagrange polynomial which consists of a companion pencil of dimensions $(n+1) s \times(n+1) s$ instead of $(n+2) s \times(n+2) s$ in [2]. We start with the following lemma.

Lemma 4.3. Suppose $\ell_{i}(\lambda)$ and $b_{i}(\lambda)$ are defined by (3.5) and (3.9), respectively. Let $p_{i}(\lambda)$ be $\ell_{i}(\lambda)$ or $b_{i}(\lambda)$, then

$$
\left(\lambda-\sigma_{i-1}\right) p_{i-1}(\lambda)=\frac{w_{i-1}}{w_{i}}\left(\lambda-\sigma_{i}\right) p_{i}(\lambda),
$$

for $i=1, \ldots, n$.

Proof. The relations between $p_{i-1}(\lambda)$ and $p_{i}(\lambda)$ follow immediately from the definitions of $\ell_{i}(\lambda)$ and $b_{i}(\lambda)$.

Theorem 4.4. Let $P(\lambda) \in \mathbb{C}^{s \times s}$ be a matrix polynomial of degree $n$ in modified Lagrange form (3.6) or in barycentric Lagrange form (3.8). Then, the $(n+1) s \times(n+1) s$ linear companion pencil

$$
L(\lambda)=C_{0}-\lambda C_{1},
$$


where

$$
C_{0}=\left[\begin{array}{ccccc}
A_{0} & A_{1} & A_{2} & \ldots & A_{n} \\
\sigma_{0} I & -\sigma_{1} \theta_{1} I & & & \\
& \sigma_{1} I & -\sigma_{2} \theta_{2} I & & \\
& & \ddots & \ddots & \\
& & & \sigma_{n-1} I & -\sigma_{n} \theta_{n} I
\end{array}\right]
$$

and

$$
C_{1}=\left[\begin{array}{ccccc}
0 & & & & \\
I & -\theta_{1} I & & & \\
& I & -\theta_{2} I & & \\
& & \ddots & \ddots & \\
& & & I & -\theta_{n} I
\end{array}\right],
$$

with $\theta_{i}=w_{i-1} / w_{i}$ for $i=1, \ldots, n$ is a linearization of $P(\lambda)$.

Again, we define

$$
\Lambda(\lambda)=\left[\begin{array}{c}
\ell_{0}(\lambda) \\
\ell_{1}(\lambda) \\
\vdots \\
\ell_{n}(\lambda)
\end{array}\right] \quad \text { and } \quad B(\lambda)=\left[\begin{array}{c}
b_{0}(\lambda) \\
b_{1}(\lambda) \\
\vdots \\
b_{n}(\lambda)
\end{array}\right],
$$

to illustrate the construction of the pencil in Theorem 4.4. Note also that $\Lambda(\lambda)=$ $\ell(\lambda) b(\lambda) B(\lambda)$. Then, similar to (4.2)-(4.3), we have for the Lagrange polynomial (3.6)

$$
\left(C_{0}-\lambda C_{1}\right)(\Lambda(\lambda) \otimes I)=e_{1} \otimes P(\lambda),
$$

and for the barycentric Lagrange polynomial (3.8)

$$
\left(C_{0}-\lambda C_{1}\right)(B(\lambda) \otimes I)=e_{1} \otimes P(\lambda),
$$

where the product of the first block row of $C_{0}-\lambda C_{1}$ with $\Lambda(\lambda) \otimes I$ and $B(\lambda) \otimes I$ is the matrix polynomial $P(\lambda)$. The remaining products simply reproduce the relations of Lemma 4.3.

Corollary 4.5. Suppose that $\left(\lambda_{\star}, x\right)$ is an eigenpair of $P(\lambda)$ and that $L(\lambda)=C_{0}-\lambda C_{1}$ is defined by Theorem 4.4. Then, evaluating (4.7) and (4.8) at $\lambda_{\star}$ and multiplying to the right by $x$ yields

$$
L\left(\lambda_{\star}\right) \cdot\left(\Lambda\left(\lambda_{\star}\right) \otimes x\right)=L\left(\lambda_{\star}\right) \cdot\left(B\left(\lambda_{\star}\right) \otimes x\right)=0 .
$$

Thus, $\lambda_{\star}$ is also an eigenvalue of $L(\lambda)=C_{0}-\lambda C_{1}$ with the corresponding structured eigenvector $\Lambda\left(\lambda_{\star}\right) \otimes x$ or $B\left(\lambda_{\star}\right) \otimes x$, since $\Lambda\left(\lambda_{\star}\right)=\ell\left(\lambda_{\star}\right) b\left(\lambda_{\star}\right) B\left(\lambda_{\star}\right)$.

Proposition 4.6. Let $C_{0}$ and $C_{1}$ be defined by (4.5) and (4.6), respectively. Then, $C_{0}-\lambda C_{1}$ is a strong linearization of

$$
\widehat{P}(\lambda):=\lambda^{n+1} 0_{s}+P(\lambda) .
$$

We will not prove this proposition, since the prove is similar to the one in [2] and the prove of the linearization in the next section. 


\subsection{Linearization of dimension $n s$}

The linearizations from $\S 4.1$ and $\S 4.2$ are not linearizations of $P(\lambda)$ but of $\underline{\widehat{P}}(\lambda)(4.4)$ and $\widehat{P}(\lambda)$ (4.9), respectively. Consequently, they contain, beside all the eigenvalues of $P(\lambda)$, also extra eigenvalues at infinity. Here, we introduce a new linearization of dimension $n s$ which results in a one-to-one mapping between the eigenstructure of the original matrix polynomial $P(\lambda)$ and the pencil $C_{0}-\lambda C_{1}$, corresponding to both finite eigenvalues and the eigenvalue at infinity.

We start by defining

$$
\tilde{\ell}_{i}(\lambda):=-\frac{\ell_{i}(\lambda)}{\lambda-\sigma_{i+1}}=-\ell(\lambda) \frac{w_{i}}{\left(\lambda-\sigma_{i}\right)\left(\lambda-\sigma_{i+1}\right)}, \quad i=0, \ldots, n-1 .
$$

Next, using (4.10) for $i=n-1$ we can rewrite $\ell_{n}(\lambda)$ as follows

$$
\ell_{n}(\lambda)=\frac{w_{n}}{w_{n-1}}\left(\sigma_{n-1}-\lambda\right) \tilde{\ell}_{n-1}(\lambda) .
$$

Then, combining (4.10) and (4.11) yields

$$
\begin{aligned}
P(\lambda) & =\sum_{i=0}^{n} A_{i} \ell_{i}(\lambda) \\
& =\sum_{i=0}^{n-1} A_{i} \ell_{i}(\lambda)+A_{n} \ell_{n}(\lambda) \\
& =\sum_{i=0}^{n-1} A_{i}\left(\sigma_{i+1}-\lambda\right) \tilde{\ell}_{i}(\lambda)+A_{n} \frac{w_{n}}{w_{n-1}}\left(\sigma_{n-1}-\lambda\right) \tilde{\ell}_{n-1}(\lambda), \\
& =\sum_{i=0}^{n-2} A_{i}\left(\sigma_{i+1}-\lambda\right) \tilde{\ell}_{i}(\lambda)+\left[A_{n-1}\left(\sigma_{n}-\lambda\right)+A_{n} \frac{w_{n}}{w_{n-1}}\left(\sigma_{n-1}-\lambda\right)\right] \tilde{\ell}_{n-1}(\lambda) .
\end{aligned}
$$

Similarly, for the barycentric Lagrange polynomial we define

$$
\widetilde{b}_{i}(\lambda):=-\frac{b_{i}(\lambda)}{\lambda-\sigma_{i+1}}=-\frac{1}{b(\lambda)} \cdot \frac{w_{i}}{\left(\lambda-\sigma_{i}\right)\left(\lambda-\sigma_{i+1}\right)}, \quad i=0, \ldots, n-1,
$$

and using (4.12) for $i=n-1$ we rewrite $b_{n}(\lambda)$ as follows

$$
b_{n}(\lambda)=\frac{w_{n}}{w_{n-1}}\left(\sigma_{n-1}-\lambda\right) \widetilde{b}_{n-1}(\lambda) .
$$

Combining (4.12) and (4.13) results in

$$
P(\lambda)=\sum_{i=0}^{n-2} A_{i}\left(\sigma_{i+1}-\lambda\right) \widetilde{b}_{i}(\lambda)+\left[A_{n-1}\left(\sigma_{n}-\lambda\right)+A_{n} \frac{w_{n}}{w_{n-1}}\left(\sigma_{n-1}-\lambda\right)\right] \widetilde{b}_{n-1}(\lambda)
$$

Before presenting the linearization, we formulate in the following lemma the relations between successive $\widetilde{\ell}_{i}(\lambda)$ and $\widetilde{b}_{i}(\lambda)$, respectively.

Lemma 4.7. Suppose $\widetilde{\ell}_{i}(\lambda)$ and $\widetilde{b}_{i}(\lambda)$ are defined by (4.10) and (4.12), respectively. Let $\widetilde{p}_{i}(\lambda)$ be $\widetilde{\ell}_{i}(\lambda)$ or $\widetilde{b}_{i}(\lambda)$, then

$$
\left(\lambda-\sigma_{i-1}\right) \tilde{p}_{i-1}(\lambda)=\frac{w_{i-1}}{w_{i}}\left(\lambda-\sigma_{i+1}\right) \tilde{p}_{i}(\lambda)
$$

for $i=1, \ldots, n-1$. 
Proof. The relations between $\widetilde{p}_{i-1}(\lambda)$ and $\widetilde{p}_{i}(\lambda)$ follow immediately from the definitions of $\widetilde{\ell}_{i}(\lambda)$ and $\widetilde{b}_{i}(\lambda)$.

Theorem 4.8. Let $P(\lambda) \in \mathbb{C}^{s \times s}$ be a matrix polynomial of degree $n$ in modified Lagrange form (3.6) or in barycentric Lagrange form (3.8). Then, the $n s \times n s$ linear companion pencil

$$
L(\lambda)=C_{0}-\lambda C_{1},
$$

where

$$
C_{0}=\left[\begin{array}{ccccc}
\sigma_{1} A_{0} & \sigma_{2} A_{1} & \cdots & \sigma_{n-1} A_{n-2} & \sigma_{n} A_{n-1}+\sigma_{n-1} \theta_{n}^{-1} A_{n} \\
\sigma_{0} I & -\sigma_{2} \theta_{1} I & & & \\
& \ddots & \ddots & & \\
& & \sigma_{n-3} I & -\sigma_{n-1} \theta_{n-2} I & \\
& & & \sigma_{n-2} I & -\sigma_{n} \theta_{n-1} I
\end{array}\right],
$$

and

$$
C_{1}=\left[\begin{array}{ccccc}
A_{0} & A_{1} & \cdots & A_{n-2} & A_{n-1}+\theta_{n}^{-1} A_{n} \\
I & -\theta_{1} I & & & \\
& \ddots & \ddots & & \\
& & I & -\theta_{n-2} I & \\
& & & I & -\theta_{n-1} I
\end{array}\right]
$$

with $\theta_{i}=w_{i-1} / w_{i}$ for $i=1, \ldots, n$ is a linearization of $P(\lambda)$.

We now define

$$
\widetilde{\Lambda}(\lambda)=\left[\begin{array}{c}
\widetilde{\ell}_{0}(\lambda) \\
\widetilde{\ell}_{1}(\lambda) \\
\vdots \\
\widetilde{\ell}_{n-1}(\lambda)
\end{array}\right] \quad \text { and } \quad \widetilde{B}(\lambda)=\left[\begin{array}{c}
\widetilde{b}_{0}(\lambda) \\
\widetilde{b}_{1}(\lambda) \\
\vdots \\
\widetilde{b}_{n-1}(\lambda)
\end{array}\right]
$$

Note that $\widetilde{\Lambda}(\lambda)=\ell(\lambda) b(\lambda) \widetilde{B}(\lambda)$. Again, we have for the Lagrange polynomial (3.6)

$$
\left(C_{0}-\lambda C_{1}\right)(\widetilde{\Lambda}(\lambda) \otimes I)=e_{1} \otimes P(\lambda),
$$

and similarly for the barycentric Lagrange polynomial (3.8)

$$
\left(C_{0}-\lambda C_{1}\right)(\widetilde{B}(\lambda) \otimes I)=e_{1} \otimes P(\lambda),
$$

where the product of the first block row of $C_{0}-\lambda C_{1}$ with $\widetilde{\Lambda}(\lambda) \otimes I$ and $\widetilde{B}(\lambda) \otimes I$ is the matrix polynomial $P(\lambda)$. The remaining products simply reproduce the relations of Lemma 4.7 .

Corollary 4.9. Suppose that $\left(\lambda_{\star}, x\right)$ is an eigenpair of $P(\lambda)$ and that $L(\lambda)=C_{0}-\lambda C_{1}$ is defined by Theorem 4.8. Then, evaluating (4.16) and (4.17) at $\lambda_{\star}$ and multiplying to the right by $x$ yields

$$
L\left(\lambda_{\star}\right) \cdot\left(\widetilde{\Lambda}\left(\lambda_{\star}\right) \otimes x\right)=L\left(\lambda_{\star}\right) \cdot\left(\widetilde{B}\left(\lambda_{\star}\right) \otimes x\right)=0 .
$$

Thus, $\lambda_{\star}$ is also an eigenvalue of $L(\lambda)=C_{0}-\lambda C_{1}$ with the corresponding structured eigenvector $\widetilde{\Lambda}\left(\lambda_{\star}\right) \otimes x$ or $\widetilde{B}\left(\lambda_{\star}\right) \otimes x$, since $\widetilde{\Lambda}\left(\lambda_{\star}\right)=\ell\left(\lambda_{\star}\right) b\left(\lambda_{\star}\right) \widetilde{B}\left(\lambda_{\star}\right)$.

We will now show that the linearization proposed in Theorem 4.8 is strong. 
Theorem 4.10. The pencil $C_{0}-\lambda C_{1}$, as defined in (4.14)-(4.15), is a strong linearization of $P(\lambda)$.

Proof. The proof consists of three parts and is similar to the one in [2]. Weak linearization is established in parts (a) and (b). Part (a) concerns eigenvalues of $P(\lambda)$ which are not equal to an interpolation point $\sigma_{i}$ for any $i$. Part (b) concerns eigenvalues, which happen to coincide with an interpolation point, and completes the proof of the weak linearization property. Part (c) shows that the linearization is strong.

Part (a). We first introduce the $n s \times n s$ block permutation matrix

$$
S:=\left[\begin{array}{ccccc}
0 & I & & & \\
0 & 0 & I & & \\
\vdots & \vdots & \ddots & \ddots & \\
0 & 0 & \cdots & 0 & I \\
I & 0 & \cdots & 0 & 0
\end{array}\right]
$$

and note that $C_{0}-\lambda C_{1}$ is a strong linearization if and only if the same is true for $S\left(C_{0}-\right.$ $\left.\lambda C_{1}\right)$. Define the $\lambda$-dependent block LU decomposition of $S\left(C_{0}-\lambda C_{1}\right)=L(\lambda) U(\lambda)$, where

$$
L(\lambda)=\left[\begin{array}{cccc}
I & & & \\
& \ddots & & \\
& & I & \\
L_{n, 1}(\lambda) & \cdots & L_{n, n-1}(\lambda) & I
\end{array}\right],
$$

with

$$
L_{n, i}(\lambda)=\sum_{k=0}^{i-1} \frac{w_{k}}{w_{i-1}} \frac{\lambda-\sigma_{i}}{\lambda-\sigma_{k}} A_{k}, \quad i=1, \ldots, n-1,
$$

and

$$
U(\lambda)=\left[\begin{array}{cccc}
-\left(\lambda-\sigma_{0}\right) I & \left(\lambda-\sigma_{2}\right) \theta_{1} I & & \\
& \ddots & \ddots & \\
& & -\left(\lambda-\sigma_{n-2}\right) I & \left(\lambda-\sigma_{n}\right) \theta_{n-1} I \\
& & & U_{n, n}(\lambda)
\end{array}\right],
$$

with

$$
U_{n, n}(\lambda)=-\frac{P(\lambda)}{w_{n-1}\left(\lambda-\sigma_{0}\right) \cdots\left(\lambda-\sigma_{n-2}\right)} .
$$

Note that $L(\lambda)$ is well-defined and nonsingular for all $\lambda \neq \sigma_{i}$ and $\operatorname{det} L(\lambda) \equiv \pm 1$. However, $U(\lambda)$ is singular at the eigenvalues of $P(\lambda)$ and, since we supposed $\lambda \neq \sigma_{i}$ in this part of the proof, all these eigenvalues are associated with $U_{n, n}(\lambda)$. Therefore, we define $\widetilde{U}(\lambda)$ to be the same as $U(\lambda)$ except for the last block entry which is replaced by

$$
\widetilde{U}_{n, n}(\lambda)=\frac{I}{w_{n-1}\left(\lambda-\sigma_{0}\right) \cdots\left(\lambda-\sigma_{n-2}\right)},
$$

then we also have $\operatorname{det} \widetilde{U}(\lambda)$ is a nonzero constant and

$$
S\left(C_{0}-\lambda C_{1}\right)=L(\lambda) U(\lambda)=L(\lambda)\left[\begin{array}{cc}
I_{(n-1) s} & 0 \\
0 & \widehat{P}(\lambda)
\end{array}\right] \widetilde{U}(\lambda) .
$$

Thus, it follows that

$$
\left[\begin{array}{cc}
I_{(n-1) s} & 0 \\
0 & \widehat{P}(\lambda)
\end{array}\right]=E(\lambda)\left(C_{0}-\lambda C_{1}\right) F(\lambda)
$$


where $E(\lambda):=L^{-1}(\lambda) S$ and $F(\lambda):=\widetilde{U}^{-1}(\lambda)$ are analytic and invertible at those eigenvalues which do not coincide with the interpolation points. For completeness, we now give the explicit forms of $E(\lambda)$ and $F(\lambda)$

$$
\begin{aligned}
& E(\lambda)=\left[\begin{array}{ccccc}
0 & I & & & \\
& 0 & I & & \\
& & \ddots & \ddots & \\
I & -L_{n, 1}(\lambda) & -L_{n, 2}(\lambda) & \cdots & -L_{n, n-1}(\lambda)
\end{array}\right], \\
& F_{i, j}(\lambda)=\left\{\begin{array}{cl}
-\frac{1}{\lambda-\sigma_{i-1}} I, & i=1, \ldots, n-1 \\
-\frac{w_{i-1}\left(\lambda-\sigma_{j}\right)}{w_{j-1}\left(\lambda-\sigma_{i-1}\right)\left(\lambda-\sigma_{i}\right)} I, & i=1, \ldots, n-2 \\
-w_{i-1} \frac{\prod_{k=0}^{n}\left(\lambda-\sigma_{k}\right)}{\left(\lambda-\sigma_{i-1}\right)\left(\lambda-\sigma_{i}\right)} I & i=1, \ldots, \ldots, n-1 \\
0 & j=n
\end{array},\right.
\end{aligned}
$$

where $L_{n, i}(\lambda), i=1, \ldots, n-1$ is defined by (4.19).

Part (b). By using the construction of equivalence transformations which are welldefined everywhere except at the nodes, part (a) of the proof shows that the partial multiplicities of all finite eigenvalues of $P(\lambda)$, with the possible exception of an eigenvalue at a interpolation point $\sigma_{i}, i=0, \ldots, n$ are reproduced in $C_{0}-\lambda C_{1}$. Now suppose that $\sigma_{i}$ is an eigenvalue of $P(\lambda)$ and also an interpolation point. Without loss of generality, we can reorder the nodes so that this node becomes $\sigma_{n}$.

We will show that the partial multiplicities of $\sigma_{n}$ in $P(\lambda)$ and $C_{0}-\lambda C_{1}$ are the same. Therefore, we return to the $\lambda$-dependent block LU decomposition of $S\left(C_{0}-\lambda C_{1}\right)=$ $L(\lambda) U(\lambda)$ and once again, we define $E(\lambda)$ and $F(\lambda)$ as in (4.21) and (4.22), respectively. Now, we observe that they are analytic in some neighbourhood of $\sigma_{n}$. Hence, the partial multiplicities of an eigenvalue, which is also an interpolation point, are the same for $P(\lambda)$ and $C_{0}-\lambda C_{1}$. Consequently, all finite eigenvalues of $P(\lambda)$ reappear in $C_{0}-\lambda C_{1}$, together with their partial multiplicities. Hence, together with part (a), this concludes the proof for $C_{0}-\lambda C_{1}$ to be a weak linearization of $P(\lambda)$.

Part (c). In order to proof the linearization is strong, we consider the reverse polynomial $P^{\#}(\lambda)$ and using Theorem 2.4, we need to show that there exist matrix functions $H(\lambda)$ and $K(\lambda)$ which are unimodular and analytic on a neighbourhood of $\lambda=0$ and for which

$$
\left[\begin{array}{cc}
I_{(n-1) s} & 0 \\
0 & P^{\#}(\lambda)
\end{array}\right]=H(\lambda)\left(\lambda C_{0}-C_{1}\right) K(\lambda) .
$$

Firstly, we return to the $\mathrm{LU}$ decomposition of $S\left(C_{0}-\lambda C_{1}\right)$ and apply the transformation $\lambda \rightarrow \lambda^{-1}$, which yields

$$
S\left(\lambda C_{0}-C_{1}\right)=\lambda L\left(\lambda^{-1}\right) U\left(\lambda^{-1}\right) .
$$

Thus, we obtain the LU factors for the reverse pencil: $S\left(\lambda C_{0}-C_{1}\right)=L_{\mathrm{rev}}(\lambda) U_{\mathrm{rev}}(\lambda)$, where

$$
L_{\mathrm{rev}}(\lambda):=L\left(\lambda^{-1}\right), \quad U_{\mathrm{rev}}(\lambda):=\lambda U\left(\lambda^{-1}\right),
$$

with $L(\lambda)$ and $U(\lambda)$ defined by (4.18) and (4.20), respectively. Note that $L_{\mathrm{rev}}(\lambda)$ is well-defined and nonsingular for all $\lambda \neq 1 / \sigma_{i}$ and $\operatorname{det} L_{\text {rev }}(\lambda) \equiv \pm 1$. Similarly to part (a), we define $\widetilde{U}_{\text {rev }}(\lambda)$ to be the same as $U_{\text {rev }}(\lambda)$ except for the last block entry which is replaced by

$$
\widetilde{U}_{\operatorname{rev} n, n}(\lambda)=-\frac{I}{w_{n-1}\left(1-\lambda \sigma_{0}\right) \cdots\left(1-\lambda \sigma_{n-2}\right)} .
$$


Thus, $\operatorname{det} \widetilde{U}_{\text {rev }}(\lambda)$ is a nonzero constant and

$$
S\left(\lambda C_{0}-C_{1}\right)=L_{\mathrm{rev}}(\lambda) U_{\mathrm{rev}}(\lambda)=L_{\mathrm{rev}}(\lambda)\left[\begin{array}{cc}
I_{(n-1) s} & 0 \\
0 & P^{\#}(\lambda)
\end{array}\right] \widetilde{U}_{\mathrm{rev}}(\lambda),
$$

and we define $H(\lambda):=\left[L_{\mathrm{rev}}(\lambda)\right]^{-1} S$ and $K(\lambda):=\left[\widetilde{U}_{\mathrm{rev}}(\lambda)\right]^{-1}$. Note that $H(\lambda)=E\left(\lambda^{-1}\right)$ and

$$
K_{i, j}(\lambda)=\left\{\begin{array}{cl}
-\frac{1}{1-\lambda \sigma_{i-1}} I, & i=1, \ldots, n-1 \\
w_{i-1}\left(1-\lambda \sigma_{j}\right) & j=i \\
-\frac{i}{w_{j-1}\left(1-\lambda \sigma_{i-1}\right)\left(1-\lambda \sigma_{i}\right)} I, & j=i+\ldots, n-2 \\
-\prod_{k=0}^{n}\left(1-\lambda \sigma_{k}\right) & i=1, \ldots, n \\
\left.-w_{i-1} \frac{\left.\lambda \sigma_{i-1}\right)\left(1-\lambda \sigma_{i}\right)}{(1-\lambda}\right) & j=n \\
0 & \text { otherwise }
\end{array}\right.
$$

In order to examine the behaviour of $S\left(\lambda C_{0}-C_{1}\right)$ near 0 , we consider the properties of $H(0)$ and $K(0)$. Since $\lim _{\lambda \rightarrow \infty} L_{n, i}(\lambda)$ is constant, with $L_{n, i}(\lambda)$ as defined by (4.19), it follows from (4.18) that $\lim _{\lambda \rightarrow 0} L\left(\lambda^{-1}\right)$ is constant too and hence $\lim _{\lambda \rightarrow 0} L^{-1}\left(\lambda^{-1}\right)$ is constant. By definition, it follows that $H(\lambda)$ is analytic and invertible at $\lambda=0$. From (4.23) we observe that $\lim _{\lambda \rightarrow 0} K(\lambda)$ is a constant upper-triangular matrix with nonzero diagonal entries. Consequently, $K(\lambda)$ is also analytic and invertible at $\lambda=0$. This completes the proof.

\section{$5 \quad$ Exploitation of pencil structure}

The companion-type matrices of the pencil $L(\lambda)=C_{0}-\lambda C_{1}$ from Theorem 4.8 are of dimension ns. Thus, as a consequence of linearization, the problem dimension is multiplied by $n$. However, in Krylov-type methods we can exploit the structure of $C_{0}$ and $C_{1}$ (4.14)-(4.15) such that we only have to deal with matrices of the original polynomial dimension $s$.

Theorem 5.1. Let $C_{0}$ and $C_{1}$ be defined by (4.14) and (4.15), respectively. Then, the linear system

$$
\left(C_{0}-\lambda C_{1}\right) x=y,
$$

with $\lambda \in \mathbb{C}$ can be efficiently solved by using only $n$ matrix-vector products of dimension $s$ and one linear system solve of dimension $s$.

Proof. Let

$$
x:=\left[\begin{array}{llll}
x_{1}^{*} & x_{2}^{*} & \cdots & x_{n}^{*}
\end{array}\right]^{*}, \quad \text { and } \quad y:=\left[\begin{array}{llll}
y_{1}^{*} & y_{2}^{*} & \cdots & y_{n}^{*}
\end{array}\right]^{*},
$$

where $x_{i}, y_{i} \in \mathbb{C}^{s}$ for $i=1, \ldots, n$. Then, the first block row of (5.1) results in

$$
\sum_{k=1}^{n}\left(\sigma_{k}-\lambda\right) A_{k-1} x_{k}+\frac{w_{n}}{w_{n-1}}\left(\sigma_{n-1}-\lambda\right) A_{n} x_{n}=y_{1}
$$

and the next block rows can be written as

$$
x_{i}=\frac{w_{i-1}}{w_{i-2}} \cdot \frac{1}{\lambda-\sigma_{i}} y_{i}+\frac{w_{i-1}}{w_{i-2}} \cdot \frac{\lambda-\sigma_{i-2}}{\lambda-\sigma_{i}} x_{i-1}, \quad i=2, \ldots, n .
$$


Now, substituting the relations (5.3) into (5.2) yields

$$
-\frac{1}{w_{0}}\left(\lambda-\sigma_{0}\right)\left(\lambda-\sigma_{1}\right)\left(\sum_{k=0}^{n} \frac{w_{k}}{\lambda-\sigma_{k}} A_{k}\right) x_{1}=y_{1}+\sum_{i=1}^{n} A_{i} \sum_{j=1}^{i} \frac{w_{i}}{w_{j-1}} \cdot \frac{\lambda-\sigma_{j}}{\lambda-\sigma_{i}} y_{j+1},
$$

with $y_{n+1}:=0$ and which can be rewritten as follows

$$
P(\lambda) x_{1}=\frac{-w_{0} \ell(\lambda)}{\left(\lambda-\sigma_{0}\right)\left(\lambda-\sigma_{1}\right)}\left(y_{1}+\sum_{i=1}^{n} A_{i} \sum_{j=1}^{i} \frac{w_{i}}{w_{j-1}} \cdot \frac{\lambda-\sigma_{j}}{\lambda-\sigma_{i}} y_{j+1}\right),
$$

or

$$
P(\lambda) x_{1}=\frac{-w_{0}}{\left(\lambda-\sigma_{0}\right)\left(\lambda-\sigma_{1}\right) b(\lambda)}\left(y_{1}+\sum_{i=1}^{n} A_{i} \sum_{j=1}^{i} \frac{w_{i}}{w_{j-1}} \cdot \frac{\lambda-\sigma_{j}}{\lambda-\sigma_{i}} y_{j+1}\right),
$$

Note that, taking into account the definition of $\ell(\lambda)$ and $b(\lambda)$, the righthand sides of (5.4) and (5.5), respectively, have polynomial dependence on $\lambda$. Thus, from (5.4) or (5.5) we can compute $x_{1}$ with only one linear system solve with $P(\lambda)$ and $n$ matrix-vector products. Next, $x_{2}, \ldots, x_{n}$ can be computed from (5.3), which completes the proof.

Note that, in case $\lambda$ is equal to one of the interpolation points $\sigma_{i}$, the linear system (5.1) has the following sparsity pattern

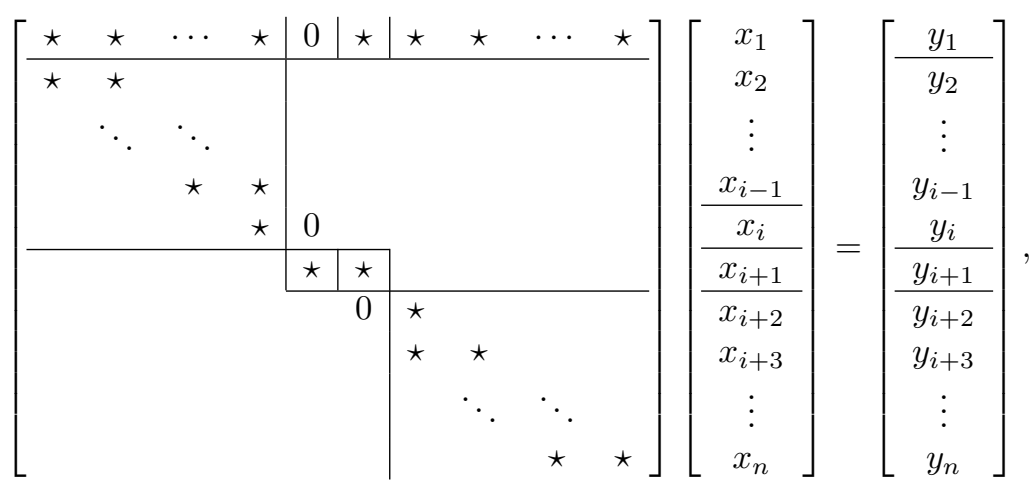

which yields a decoupling. The system can now be solved by using forward and backward substitution. Again, we only need $n$ matrix-vector products and one linear system solve, both of dimension $s$.

\section{Hermite interpolation}

In the previous sections, we considered interpolating matrix polynomials in distinct points. From here on, we also allow multiple interpolation. We start with reviewing the Hermite interpolating Lagrange and barycentric matrix polynomial. The next section discusses its corresponding linearizations.

\subsection{Lagrange Hermite form}

We still suppose that $\sigma_{i}, i=0, \ldots, n$ are $n+1$ distinct interpolation points, but now with corresponding multiplicities $m_{i}$, with

$$
m_{0}+\cdots+m_{n}=N+1,
$$


where $N$ is the degree of the corresponding interpolating polynomial $P(\lambda)$. The Lagrange form can now be generalized to multiple interpolation by

$$
P(\lambda)=\sum_{i=0}^{n} \sum_{j=0}^{m_{i}-1} \frac{A_{i}^{(j)}}{j !} \ell_{i, j}(\lambda)
$$

where

$$
\ell_{i, j}(\lambda)=\ell(\lambda) \sum_{k=j}^{m_{i}-1} \frac{w_{i, k}}{\left(\lambda-\sigma_{i}\right)^{k-j+1}}
$$

is the generalization of (3.5) for multiple interpolation with

$$
\ell(\lambda)=\left(\lambda-\sigma_{0}\right)^{m_{0}}\left(\lambda-\sigma_{1}\right)^{m_{1}} \cdots\left(\lambda-\sigma_{n}\right)^{m_{n}},
$$

the generalization of (3.3). The constants $w_{i, j}$ are called the generalized barycentric weights. For the computation of these $w_{i, k}$ we refer to $[4,13]$. Similar to $(3.6)$, we can bring the factor $\ell(\lambda)$ in front of the sums, yielding

$$
P(\lambda)=\ell(\lambda) \sum_{i=0}^{n} \sum_{j=0}^{m_{i}-1} \frac{A_{i}^{(j)}}{j !} \sum_{k=j}^{m_{i}-1} \frac{w_{i, k}}{\left(\lambda-\sigma_{i}\right)^{k+1}} .
$$

\subsection{Barycentric Hermite form}

The barycentric interpolating matrix polynomial for multiple interpolation can be obtained in a similar way as in Section 3.3. Again, we start from

$$
1=\ell(\lambda) \sum_{i=0}^{n} \sum_{j=0}^{m_{i}-1} \frac{w_{i, j}}{\left(\lambda-\sigma_{i}\right)^{j+1}} .
$$

Dividing the Lagrange Hermite form (6.2) by (6.3) and cancelling out the common factor $\ell(\lambda)$, we obtain the barycentric Hermite form:

$$
P(\lambda)=\frac{\sum_{i=0}^{n} \sum_{j=0}^{m_{i}-1} \frac{A_{i}^{(j)}}{j !} \sum_{k=j}^{m_{i}-1} \frac{w_{i, k}}{\left(\lambda-\sigma_{i}\right)^{k+1}}}{\sum_{i=0}^{n} \sum_{j=0}^{m_{i}-1} \frac{w_{i, j}}{\left(\lambda-\sigma_{i}\right)^{j+1}}}=\sum_{i=0}^{n} \sum_{j=0}^{m_{i}-1} \frac{A_{i}^{(j)}}{j !} b_{i, j}(\lambda),
$$

where

$$
b_{i, j}(\lambda)=\frac{1}{b(\lambda)} \sum_{k=j}^{m_{i}-1} \frac{w_{i, k}}{\left(\lambda-\sigma_{i}\right)^{k-j+1}}, \quad i=0, \ldots, n, \quad j=0, \ldots, m_{i}-1,
$$

with

$$
b(\lambda)=\sum_{i=0}^{n} \sum_{j=0}^{m_{i}-1} \frac{w_{i, j}}{\left(\lambda-\sigma_{i}\right)^{j+1}}
$$

\section{Linearization of the Lagrange and barycentric Her- mite polynomial}

The linearization of the Lagrange polynomial by Amiraslani [1] was generalized for multiple interpolation by Shakoori [14]. We review this linearization for the Hermite 
Lagrange and barycentric Hermite matrix polynomial. Next, we generalize our new linearization of $\S 4.3$ for which there is again a one-to-one correspondence between the eigenvalues of the original matrix polynomial $P(\lambda)$ and the ones of the companion pencil $C_{0}-\lambda C_{1}$.

\subsection{Linearization of dimension $(N+2) s$}

The companion pencil of the barycentric Hermite polynomial was introduced in [14, 5]. Here, we review this linearization for matrix polynomials in a slightly different form by the following theorem.

Theorem 7.1. Let $P(\lambda) \in \mathbb{C}^{s \times s}$ be a matrix polynomial of degree $N$ in Lagrange Hermite form (6.2) or in barycentric Hermite form (6.4). Then, the $(N+2) s \times(N+2) s$ linear companion pencil

$$
L(\lambda)=\mathbf{C}_{0}-\lambda \mathbf{C}_{1},
$$

where

$$
\mathbf{C}_{0}=\left[\begin{array}{ccccc}
\mathbf{A}_{0} & \mathbf{A}_{1} & \cdots & \mathbf{A}_{n} & 0 \\
\mathbf{J}_{0} & & & & \mathbf{W}_{0} \\
& \mathbf{J}_{1} & & & \mathbf{W}_{1} \\
& & \ddots & & \vdots \\
& & & \mathbf{J}_{n} & \mathbf{W}_{n}
\end{array}\right], \quad \mathbf{C}_{1}=\left[\begin{array}{ccccc}
0 & & & & \\
I & 0 & & & \\
& I & 0 & & \\
& & \ddots & \ddots & \\
& & I & 0
\end{array}\right],
$$

with

$$
\begin{aligned}
& \mathbf{A}_{i}=\left[\begin{array}{llll}
\frac{A_{i}^{(0)}}{0 !} & \frac{A_{i}^{(1)}}{1 !} & \cdots & \frac{A_{i}^{\left(m_{i}-1\right)}}{\left(m_{i}-1\right) !}
\end{array}\right] \in \mathbb{C}^{s \times m_{i} s}, \quad i=0, \ldots, n, \\
& \mathbf{W}_{i}=\left[\begin{array}{c}
w_{i, 0} I \\
w_{i, 1} I \\
\vdots \\
w_{i, m_{i}-1} I
\end{array}\right] \in \mathbb{C}^{m_{i} s \times s}, \quad i=0, \ldots, n, \\
& \mathbf{J}_{i}=\left[\begin{array}{cccc}
\sigma_{i} I & I & & \\
& \ddots & \ddots & \\
& & \sigma_{i} I & I \\
& & & \sigma_{i} I
\end{array}\right] \in \mathbb{C}^{m_{i} s \times m_{i} s}, \quad i=0, \ldots, n,
\end{aligned}
$$

is a linearization of $P(\lambda)$.

In order to illustrate the construction of the pencil in Theorem 7.1, we define $\underline{\boldsymbol{\Lambda}}(\lambda)$ and $\underline{\mathbf{B}}(\lambda)$ as follows

$$
\underline{\boldsymbol{\Lambda}}(\lambda)=\left[\begin{array}{c}
\boldsymbol{\ell}_{0}(\lambda) \\
\boldsymbol{\ell}_{1}(\lambda) \\
\vdots \\
\boldsymbol{\ell}_{n}(\lambda) \\
\ell(\lambda)
\end{array}\right] \quad \text { and } \quad \underline{\mathbf{B}}(\lambda)=\left[\begin{array}{c}
\mathbf{b}_{0}(\lambda) \\
\mathbf{b}_{1}(\lambda) \\
\vdots \\
\mathbf{b}_{n}(\lambda) \\
b(\lambda)
\end{array}\right]
$$

respectively, where

$$
\ell_{i}(\lambda)=\left[\begin{array}{c}
\ell_{i, 0}(\lambda) \\
\ell_{i, 1}(\lambda) \\
\vdots \\
\ell_{i, m_{i}-1}(\lambda)
\end{array}\right] \quad \text { and } \quad \mathbf{b}_{i}(\lambda)=\left[\begin{array}{c}
b_{i, 0}(\lambda) \\
b_{i, 1}(\lambda) \\
\vdots \\
b_{i, m_{i}-1}(\lambda)
\end{array}\right]
$$


for $i=0, \ldots, n$. Note that $\underline{\boldsymbol{\Lambda}}(\lambda)=\ell(\lambda) b(\lambda) \underline{\mathbf{B}}(\lambda)$. Then, we have for the Hermite Lagrange polynomial (6.2)

$$
\left(\mathbf{C}_{0}-\lambda \mathbf{C}_{1}\right)(\underline{\boldsymbol{\Lambda}}(\lambda) \otimes I)=e_{1} \otimes P(\lambda),
$$

and for the barycentric Hermite polynomial (6.4)

$$
\left(\mathbf{C}_{0}-\lambda \mathbf{C}_{1}\right)(\underline{\mathbf{B}}(\lambda) \otimes I)=e_{1} \otimes P(\lambda),
$$

where the product of the first $s$ rows of $\mathbf{C}_{0}-\lambda \mathbf{C}_{1}$ with $\underline{\boldsymbol{\Lambda}}(\lambda) \otimes I$ and $\underline{\mathbf{B}}(\lambda) \otimes I$ is the matrix polynomial $P(\lambda)$ and the remaining products simply reproduce the relations (6.1) and (6.5), respectively.

Corollary 7.2. Suppose that $\left(\lambda_{\star}, x\right)$ is an eigenpair of $P(\lambda)$ and that $L(\lambda)=\mathbf{C}_{0}-\lambda \mathbf{C}_{1}$ is defined by Theorem 7.1. Then, evaluating (7.6) and (7.7) at $\lambda_{\star}$ and multiplying to the right by $x$ yields

$$
L\left(\lambda_{\star}\right) \cdot\left(\underline{\boldsymbol{\Lambda}}\left(\lambda_{\star}\right) \otimes x\right)=L\left(\lambda_{\star}\right) \cdot\left(\underline{\mathbf{B}}\left(\lambda_{\star}\right) \otimes x\right)=0 .
$$

Thus, $\lambda_{\star}$ is also an eigenvalue of $L(\lambda)=\mathbf{C}_{0}-\lambda \mathbf{C}_{1}$ with the corresponding structured eigenvector $\underline{\boldsymbol{\Lambda}}\left(\lambda_{\star}\right) \otimes x$ or $\underline{\mathbf{B}}\left(\lambda_{\star}\right) \otimes x$, since $\underline{\boldsymbol{\Lambda}}\left(\lambda_{\star}\right)=\ell\left(\lambda_{\star}\right) b\left(\lambda_{\star}\right) \underline{\mathbf{B}}\left(\lambda_{\star}\right)$.

\subsection{Linearization of dimension $\mathrm{Ns}$}

We now propose a new linearization for the Hermite Lagrange and barycentric Hermite polynomial which consists of a companion pencil of dimensions $N s \times N s$ instead of $(N+2) s \times(N+2) s$.

Similar to $\S 4.3$, we use $\widetilde{p}_{i, j}(\lambda)$ to denote

$$
\begin{array}{rlrl}
\widetilde{p}_{i, j}(\lambda):=-\frac{p_{i, j}(\lambda)}{\lambda-\sigma_{i+1}}, & i & =0, \ldots, n-1 \\
& j=0, \ldots, m_{i}-1 \\
\widetilde{p}_{n, j}(\lambda):=-\frac{p_{n, j}(\lambda)}{\lambda-\sigma_{n-1}}, & j & =0, \ldots, m_{i}-2,
\end{array}
$$

where $p_{i, j}(\lambda)$ is $\ell_{i, j}(\lambda)$ or $b_{i, j}(\lambda)$. Next, using (7.8) we can rewrite $p_{n, m_{n}-1}(\lambda)$ as follows

$$
p_{n, m_{n}-1}(\lambda)=\frac{w_{n, m_{n}-1}}{w_{n-1, m_{n-1}-1}}\left(\sigma_{n-1}-\lambda\right) \widetilde{p}_{n-1, m_{n-1}-1}(\lambda),
$$

Then, combining (7.8) and (7.9) yields

$$
\begin{aligned}
P(\lambda)= & \sum_{i=0}^{n} \sum_{j=0}^{m_{i}-1} \frac{A_{i}^{(j)}}{j !} p_{i, j}(\lambda), \\
= & \sum_{i=0}^{n-1} \sum_{j=0}^{m_{i}-1} \frac{A_{i}^{(j)}}{j !} p_{i, j}(\lambda)+\sum_{j=0}^{m_{n}-2} \frac{A_{n}^{(j)}}{j !} p_{n, j}(\lambda)+\frac{A_{n}^{\left(m_{n}-1\right)}}{\left(m_{n}-1\right) !} p_{n, m_{n}-1}(\lambda), \\
= & \sum_{i=0}^{n-1} \sum_{j=0}^{m_{i}-1} \frac{A_{i}^{(j)}}{j !}\left(\sigma_{i+1}-\lambda\right) \widetilde{p}_{i, j}(\lambda)+\sum_{j=0}^{m_{n}-2} \frac{A_{n}^{(j)}}{j !}\left(\sigma_{n-1}-\lambda\right) \widetilde{p}_{n, j}(\lambda) \\
& \quad+\frac{A_{n}^{\left(m_{n}-1\right)}}{\left(m_{n}-1\right) !} \frac{w_{n, m_{n}-1}}{w_{n-1, m_{n-1}-1}}\left(\sigma_{n-1}-\lambda\right) \widetilde{p}_{n-1, m_{n-1}-1}(\lambda),
\end{aligned}
$$




$$
\begin{aligned}
= & \sum_{i=0}^{n-2} \sum_{j=0}^{m_{i}-1} \frac{A_{i}^{(j)}}{j !}\left(\sigma_{i+1}-\lambda\right) \widetilde{p}_{i, j}(\lambda)+\sum_{j=0}^{m_{n-1}-2} \frac{A_{n-1}^{(j)}}{j !}\left(\sigma_{n}-\lambda\right) \widetilde{p}_{n-1, j}(\lambda) \\
& +\left[\frac{A_{n-1}^{\left(m_{n-1}-1\right)}}{\left(m_{n-1}-1\right) !}\left(\sigma_{n}-\lambda\right)+\frac{A_{n}^{\left(m_{n}-1\right)}}{\left(m_{n}-1\right) !} \frac{w_{n, m_{n}-1}}{w_{n-1, m_{n-1}-1}}\left(\sigma_{n-1}-\lambda\right)\right] \widetilde{p}_{n-1, m_{n-1}-1}(\lambda), \\
& +\sum_{j=0}^{m_{n}-2} \frac{A_{n}^{(j)}}{j !}\left(\sigma_{n-1}-\lambda\right) \widetilde{p}_{n, j}(\lambda) .
\end{aligned}
$$

Before presenting the linearization, we formulate the relations between successive $\widetilde{\ell}_{i, j}(\lambda)$ and $\widetilde{b}_{i, j}(\lambda)$, respectively, in the following lemma.

Lemma 7.3. Suppose that $\widetilde{p}_{i, j}(\lambda)$ is $\widetilde{\ell}_{i, j}(\lambda)$ or $\widetilde{b}_{i, j}(\lambda)$, defined by (7.8). Then, we have the following relations

$$
\begin{aligned}
\left(\lambda-\sigma_{i}\right) \widetilde{p}_{i, 0}(\lambda) & =\widetilde{p}_{i, 1}(\lambda)+\frac{w_{i, 0}}{w_{i, m_{i}-1}}\left(\lambda-\sigma_{i}\right) \widetilde{p}_{i, m_{i}-1}(\lambda), \\
\left(\lambda-\sigma_{i}\right) \widetilde{p}_{i, 1}(\lambda) & =\widetilde{p}_{i, 2}(\lambda)+\frac{w_{i, 1}}{w_{i, m_{i}-1}}\left(\lambda-\sigma_{i}\right) \widetilde{p}_{i, m_{i}-1}(\lambda), \\
& \vdots \\
\left(\lambda-\sigma_{i}\right) \widetilde{p}_{i, m_{i}-2}(\lambda) & =\widetilde{p}_{i, m_{i}-1}(\lambda)+\frac{w_{i, m_{i}-2}}{w_{i, m_{i}-1}}\left(\lambda-\sigma_{i}\right) \widetilde{p}_{i, m_{i}-1}(\lambda),
\end{aligned}
$$

for $i=0, \ldots, n-1$ and

$$
\left(\lambda-\sigma_{i-1}\right) \widetilde{p}_{i-1, m_{i-1}-1}(\lambda)=\frac{w_{i-1, m_{i-1}-1}}{w_{i, m_{i}-1}}\left(\lambda-\sigma_{i+1}\right) \widetilde{p}_{i, m_{i}-1}(\lambda),
$$

for $i=1, \ldots, n-2$. We also have

$$
\begin{aligned}
\left(\lambda-\sigma_{n}\right) \widetilde{p}_{n, 0}(\lambda) & =\widetilde{p}_{n, 1}(\lambda)+\frac{w_{n, 0}}{w_{n-1, m_{n-1}-1}}\left(\lambda-\sigma_{n}\right) \widetilde{p}_{n-1, m_{n-1}-1}(\lambda), \\
& \vdots \\
\left(\lambda-\sigma_{n}\right) \widetilde{p}_{n, m_{n}-3}(\lambda) & =\widetilde{p}_{n, m_{n}-2}(\lambda)+\frac{w_{n, m_{n}-3}}{w_{n-1, m_{n-1}-1}}\left(\lambda-\sigma_{n}\right) \widetilde{p}_{n-1, m_{n-1}-1}(\lambda), \\
\left(\lambda-\sigma_{n}\right) \widetilde{p}_{n, m_{n}-2}(\lambda) & =\left(\frac{w_{n, m_{n}-1}}{w_{n-1, m_{n-1}-1}}+\frac{w_{n, m_{n}-2}}{w_{n-1, m_{n-1}-1}}\left(\lambda-\sigma_{n}\right)\right) \widetilde{p}_{n-1, m_{n-1}-1}(\lambda) .
\end{aligned}
$$

Proof. These relations follow immediately from the definitions $(7.8)$ of $\widetilde{\ell}_{i, j}(\lambda)$ and $\widetilde{b}_{i, j}(\lambda)$ and the relation (7.9).

Theorem 7.4. Let $P(\lambda) \in \mathbb{C}^{n \times n}$ be a matrix polynomial of degree $N$ in Lagrange Hermite form (6.2) or in barycentric Hermite form (6.4). Then, the $N s \times N$ s linear companion pencil

$$
L(\lambda)=\mathbf{C}_{0}-\lambda \mathbf{C}_{1},
$$


where

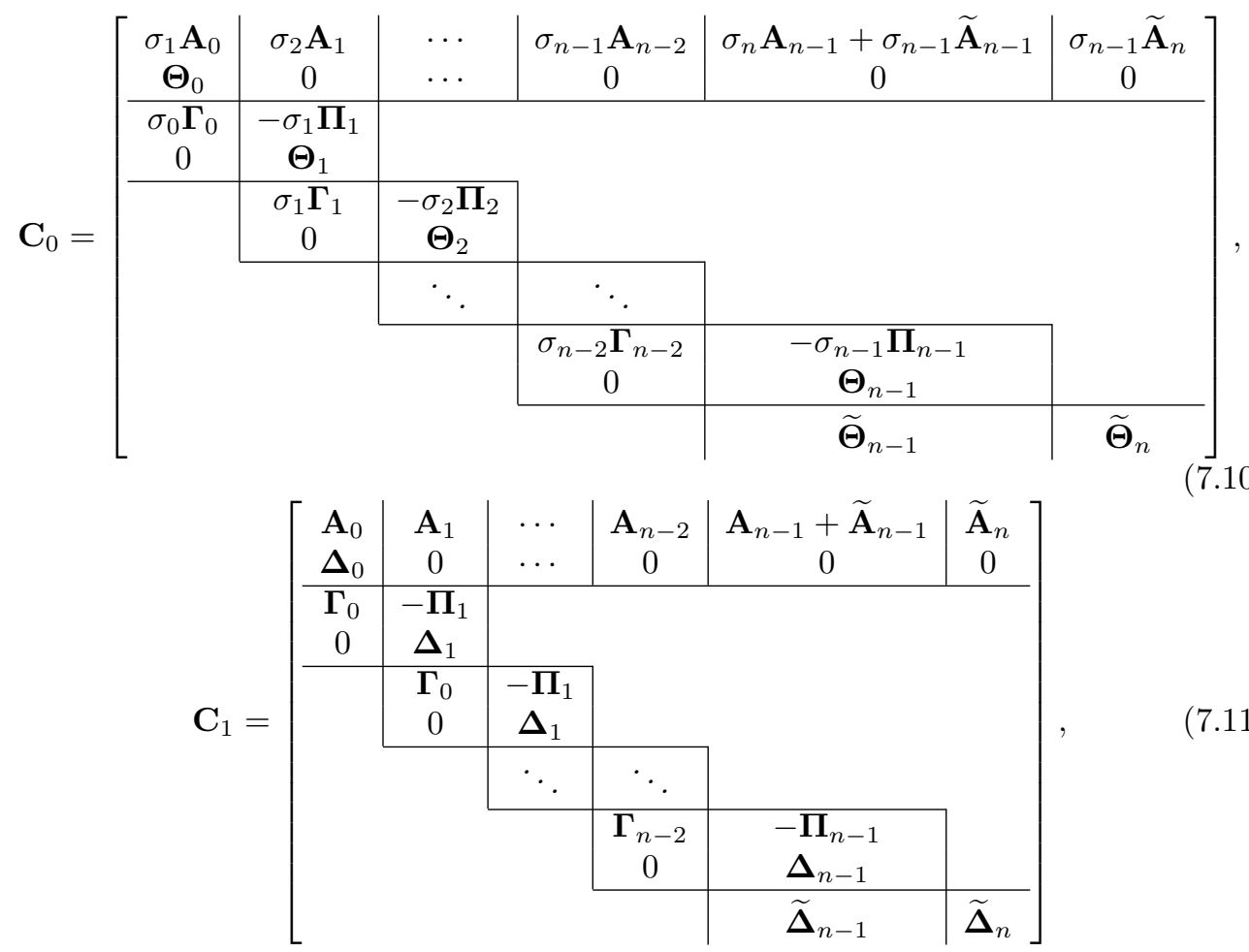

with $\mathbf{A}_{i}, i=0,1, \ldots, n-1$ as defined by (7.2) and

$$
\begin{aligned}
& \mathbf{A}_{i}=\left[\begin{array}{cccc}
\frac{A_{i}^{(0)}}{0 !} & \frac{A_{i}^{(1)}}{1 !} & \cdots & \frac{A_{i}^{\left(m_{i}-1\right)}}{\left(m_{i}-1\right) !}
\end{array}\right], \quad i=0, \ldots, n-1, \\
& \boldsymbol{\Pi}_{i}=\left[\begin{array}{llll}
0 & \cdots & 0 & \frac{w_{i-1, m_{i-1}-1}}{w_{i, m_{i}-1}} I
\end{array}\right], \quad i=1, \ldots, n-1, \\
& \boldsymbol{\Gamma}_{i}=\left[\begin{array}{llll}
0 & \cdots & 0 & I
\end{array}\right], \quad i=0, \ldots, n-2, \\
& \boldsymbol{\Theta}_{i}=\left[\begin{array}{ccccc}
\sigma_{i} I & I & & & -\sigma_{i} \frac{w_{i, 0}}{w_{i, m_{i}-1}} I \\
& \ddots & \ddots & & \vdots \\
& & \sigma_{i} I & I & -\sigma_{i} \frac{w_{i, m_{i}-3}}{w_{i, m_{i}-1}} I \\
& & & \sigma_{i} I & \left(1-\sigma_{i} \frac{w_{i, m_{i}-2}}{w_{i, m_{i}-1}}\right) I
\end{array}\right], \quad i=0, \ldots, n-1, \\
& \boldsymbol{\Delta}_{i}=\left[\begin{array}{ccccc}
I & & & & -\frac{w_{i, 0}}{w_{i, m_{i}-1}} I \\
& \ddots & & & \vdots \\
& & & & -\frac{w_{i, m_{i}-3}}{w_{i, m_{i}-1}} I \\
& & & I & -\frac{w_{i, m_{i}-2}}{w_{i, m_{i}-1}} I
\end{array}\right], \quad i=0, \ldots, n-1,
\end{aligned}
$$

where $\mathbf{A}_{i}, \boldsymbol{\Gamma}_{i}, \boldsymbol{\Pi}_{i} \in \mathbb{C}^{s \times m_{i} s}$ and $\boldsymbol{\Theta}_{i}, \boldsymbol{\Delta}_{i} \in \mathbb{C}^{\left(m_{i}-1\right) s \times m_{i} s}$ and

$$
\begin{aligned}
\widetilde{\mathbf{A}}_{n-1} & =\left[\begin{array}{llll}
0 & \cdots & 0 & \frac{w_{n, m_{n}-1}}{w_{n-1, m_{n-1}-1}} \frac{A_{n}^{\left(m_{n}-1\right)}}{\left(m_{n}-1\right) !}
\end{array}\right] \in \mathbb{C}^{s \times m_{n-1} s}, \\
\widetilde{\mathbf{A}}_{n} & =\left[\begin{array}{llll}
\frac{A_{n}^{(0)}}{0 !} & \frac{A_{n}^{(1)}}{1 !} & \cdots & \frac{A_{n}^{\left(m_{n}-2\right)}}{\left(m_{n}-2\right) !}
\end{array}\right] \in \mathbb{C}^{s \times\left(m_{n}-1\right) s},
\end{aligned}
$$




$$
\begin{aligned}
& \widetilde{\boldsymbol{\Theta}}_{n-1}=\left[\begin{array}{cccc}
0 & & & -\sigma_{n} \frac{w_{n, 0}}{w_{n-1, m_{n-1}-1}} I \\
& \ddots & & \vdots \\
& & 0 & -\sigma_{n} \frac{w_{n, m_{n}-3}}{w_{n-1, m_{n-1}-1}} I \\
& & 0 & \left(\frac{w_{n, m_{n}-1}}{w_{n-1, m_{n-1}-1}}-\sigma_{n} \frac{w_{n, m}-2}{w_{n-1, m_{n-1}-1}}\right) I
\end{array}\right] \in \mathbb{C}^{\left(m_{n}-1\right) s \times m_{n} s}, \\
& \widetilde{\boldsymbol{\Theta}}_{n}=\left[\begin{array}{cccc}
\sigma_{n} I & I & & \\
& \ddots & \ddots & \\
& & \sigma_{n} I & I \\
& & & \sigma_{n} I
\end{array}\right] \in \mathbb{C}^{\left(m_{n}-1\right) s \times\left(m_{n}-1\right) s},
\end{aligned}
$$

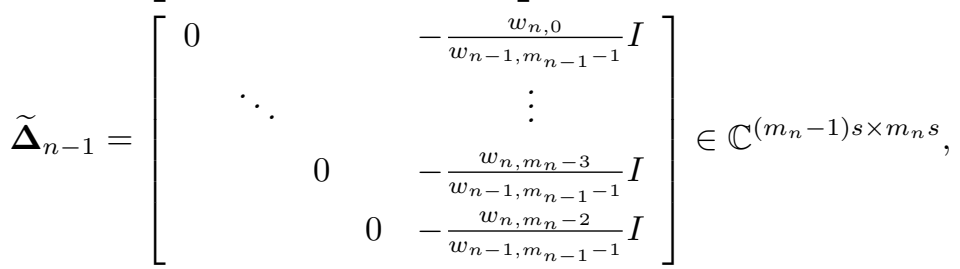

$$
\begin{aligned}
& \widetilde{\boldsymbol{\Delta}}_{n}=I \in \mathbb{C}^{\left(m_{n}-1\right) s \times\left(m_{i}-1\right) s},
\end{aligned}
$$

is a linearization of $P(\lambda)$.

We now define

$$
\widetilde{\boldsymbol{\Lambda}}(\lambda)=\left[\begin{array}{c}
\widetilde{\mathbf{l}}_{0}(\lambda) \\
\widetilde{\mathbf{l}}_{1}(\lambda) \\
\vdots \\
\widetilde{\mathbf{l}}_{n}(\lambda)
\end{array}\right] \quad \text { and } \quad \widetilde{\mathbf{B}}(\lambda)=\left[\begin{array}{c}
\widetilde{\mathbf{b}}_{0}(\lambda) \\
\widetilde{\mathbf{b}}_{1}(\lambda) \\
\vdots \\
\widetilde{\mathbf{b}}_{n}(\lambda)
\end{array}\right]
$$

where

$$
\widetilde{\mathbf{l}}_{i}(\lambda)=\left[\begin{array}{c}
\tilde{\ell}_{i, 0}(\lambda) \\
\widetilde{\ell}_{i, 1}(\lambda) \\
\vdots \\
\widetilde{\ell}_{i, k_{i}}(\lambda)
\end{array}\right] \quad \text { and } \quad \widetilde{\mathbf{b}}_{i}(\lambda)=\left[\begin{array}{c}
\widetilde{b}_{i, 0}(\lambda) \\
\widetilde{b}_{i, 1}(\lambda) \\
\vdots \\
\widetilde{b}_{i, k_{i}}(\lambda)
\end{array}\right]
$$

for $i=0,1, \ldots, n$ with $k_{i}=m_{i}-1$ for $i=0,1, \ldots, n-1$ and $k_{n}=m_{n}-2$. Note also that $\widetilde{\boldsymbol{\Lambda}}(\lambda)=\ell(\lambda) b(\lambda) \widetilde{\mathbf{B}}(\lambda)$. Again, we have for the Hermite Lagrange form (6.2)

$$
\left(\mathbf{C}_{0}-\lambda \mathbf{C}_{1}\right)(\widetilde{\boldsymbol{\Lambda}}(\lambda) \otimes I)=e_{1} \otimes P(\lambda),
$$

and similarly for the barycentric Hermite form (6.4)

$$
\left(\mathbf{C}_{0}-\lambda \mathbf{C}_{1}\right)(\widetilde{\mathbf{B}}(\lambda) \otimes I)=e_{1} \otimes P(\lambda),
$$

where the product of the first $s$ rows of $\mathbf{C}_{0}-\lambda \mathbf{C}_{1}$ with $\widetilde{\mathbf{\Lambda}}(\lambda) \otimes I$ and $\widetilde{\mathbf{B}}(\lambda) \otimes I$ is the matrix polynomial $P(\lambda)$. The remaining products reproduce the relations of Lemma 7.3.

Corollary 7.5. Suppose that $\left(\lambda_{\star}, x\right)$ is an eigenpair of $P(\lambda)$ and that $L(\lambda)=\mathbf{C}_{0}-\lambda \mathbf{C}_{1}$ is defined by Theorem 7.4. Then, evaluating (7.12) and (7.13) at $\lambda_{\star}$ and multiplying to the right by $x$ yields

$$
L\left(\lambda_{\star}\right) \cdot\left(\widetilde{\mathbf{\Lambda}}\left(\lambda_{\star}\right) \otimes x\right)=L\left(\lambda_{\star}\right) \cdot\left(\widetilde{\mathbf{B}}\left(\lambda_{\star}\right) \otimes x\right)=0 .
$$

Thus, $\lambda_{\star}$ is also an eigenvalue of $L(\lambda)=\mathbf{C}_{0}-\lambda \mathbf{C}_{1}$ with the corresponding structured eigenvector $\widetilde{\boldsymbol{\Lambda}}\left(\lambda_{\star}\right) \otimes x$ or $\widetilde{\mathbf{B}}\left(\lambda_{\star}\right) \otimes x$, since $\widetilde{\boldsymbol{\Lambda}}\left(\lambda_{\star}\right)=\ell\left(\lambda_{\star}\right) b\left(\lambda_{\star}\right) \widetilde{\mathbf{B}}\left(\lambda_{\star}\right)$. 
Proposition 7.6. The pencil $\mathbf{C}_{0}-\lambda \mathbf{C}_{1}$, as defined in (7.10)-(7.11), is a strong linearization of $P(\lambda)$.

Proof. The proof is similar to the one of Theorem 4.8 .

\section{Conclusions}

In this paper, we introduced two new and more compact linearizations for interpolating Lagrange and barycentric Lagrange matrix polynomials $P(\lambda)$. For the proposed linearization of dimension $n s$ there is a one-to-one correspondence between the eigenpairs of $P(\lambda)$ and the eigenpairs of the pencil such that no extra eigenvalues at infinity are introduced any more. We proved that this linearization is strong. Moreover, the structure of the companion-type matrices can be exploited such that in Krylov-type methods only $n$ matrix-vector products and one linear system solve, both of dimension $s$, are required. We also generalized for multiple interpolation and introduced new linearizations for Hermite Lagrange and barycentric Hermite matrix polynomials.

We have implemented the linearizations and the exploitation of the pencil structure in Matlab. The codes can be downloaded from http://people.cs.kuleuven.be/ roel. vanbeeumen/software/lin-lagr.html.

\section{References}

[1] A. Amiraslani, New algorithms for matrices, polynomials and matrix polynomials, PhD thesis, University of Western Ontario, London, Ontario, 2006.

[2] A. Amiraslani, R. M. Corless, and P. Lancaster, Linearization of matrix polynomials expressed in polynomial bases, IMA J. Numer. Anal., 29 (2009), pp. $141-157$.

[3] J.-P. Berrut And L. N. Trefethen, Barycentric Lagrange Interpolation, SIAM Rev., 46 (2004), pp. 501-517.

[4] J. Butcher, R. M. Corless, L. Gonzalez-Vega, And A. Shakoori, Polynomial algebra for Birkhoff interpolants, Numer. Algorithms, 56 (2011), pp. 319-347.

[5] R. M. Corless, A. Shakoori, D. A. Aruliah, And L. Gonzalez-Vega, Barycentric Hermite Interpolants for Event Location in Initial-Value Problems, JNAIAM. J. Numer. Anal. Ind. Appl. Math., 3 (2008), pp. 1-16.

[6] C. EfFenberger And D. Kressner, Chebyshev interpolation for nonlinear eigenvalue problems, BIT, 52 (2012), pp. 933-951.

[7] I. Gohberg, P. Lancaster, And L. Rodman, Matrix Polynomials, Academic Press, New York, 1982.

[8] N. J. Higham, The numerical stability of barycentric Lagrange interpolation, IMA J. Numer. Anal., 24 (2004), pp. 547-556.

[9] E. Jarlebring, W. Michiels, and K. Meerbergen, A linear eigenvalue algorithm for the nonlinear eigenvalue problem, Numer. Math., 122 (2012), pp. 169-195.

[10] J. L. LAGRANGE, Leçons élémentaires sur les mathématiques données à l'École Normale en 1795, in Oeuvres de Lagrange VII, Gauthier-Villars, Paris, 1877, pp. $183-287$. 
[11] P. LANCASTER, Linearization of regular matrix polynomials, Electron. J. Linear Algebra, 17 (2008), pp. 21-27.

[12] D. S. Mackey, N. Mackey, C. Mehl, and V. Mehrmann, Vector Spaces of Linearizations for Matrix Polynomials, SIAM J. Matrix Anal. Appl., 28 (2006), pp. $971-1004$.

[13] B. Sadiq And D. Viswanath, Barycentric Hermite Interpolation, SIAM J. Sci. Comput., 35 (2013), pp. A1254-A1270.

[14] A. Shakoori, Bivariate Polynomial Solver by Values, PhD thesis, University of Western Ontario, London, Ontario, 2007.

[15] R. Van Beeumen, K. Meerbergen, And W. Michiels, A Rational Krylov Method Based on Hermite Interpolation for Nonlinear Eigenvalue Problems, SIAM J. Sci. Comput., 35 (2013), pp. A327-A350. 